\title{
Aufsuchende Soziale Arbeit als sozialräumliche und lebensweltorientierte Schadensminderung
}

Arbeitsfeld Aufsuchende Soziale Arbeit

Mathias Arbogast ${ }^{1}$

\section{Herausbildung der aufsuchenden Sozialen Arbeit als eigenständi- ges Arbeitsfeld innerhalb der Sozialen Arbeit}

In ihrem Selbstverständnis ist aufsuchende Soziale Arbeit (aSa) professionelles Handeln im öffentlichen bzw. halböffentlichen Raum - d. h. sie vertritt klare Haltungen und handelt methodisch. Doch der Weg hin zu einem eigenständigen, reflektierten Arbeitsfeld innerhalb der Sozialen Arbeit war weit.

\subsection{Erste Theoriebildung und Praxisprojekte}

Eine erste systematisch-theoretische Grundlegung der aSa lieferte die sog. sozialökonomische Chicagoer Schule in den 1920er-Jahren. Am damaligen Lehrstuhl für Soziologie und Anthropologie an der University of Chicago entstanden wegweisende Publikationen in den Bereichen der Stadt-, Milieu- und Devianzsoziologie, auf deren Basis sog. "area workers» in bestimmten, als problematisch kategorisierten Stadtteilen tätig wurden. Losgelöst von ihren angestammten Einrichtungen wandten sich die «area workers» vorrangig delinquierenden Jugendgangs zu (vgl. Specht 2010: 44-47). Der Fokus lag dabei auf der Resozialisierung bzw. der Kriminalprävention und nicht auf deren Zerschlagung (vgl. Steffan 2007: 948f.). In der Folge entstanden die ersten Streetwork-Projekte, unter Einbezug des örtlichen Gemeinwesens und unter Berücksichtigung des Gruppenkontextes (vgl. ebd.). Für den deutschen Sprachraum finden sich ähnliche An-

\footnotetext{
${ }^{1}$ Leiter Fachstelle 'Sozialarbeit', Katholische Kirche Region Bern. Ehem. Leiter 'aufsuchende Soziale Arbeit' und 'Schalter 20', Verein Kirchliche Gassenarbeit Luzern (bis Juni 2019).
} 
sätze für die Zeit der Weimarer Republik, in der sich Pastoren und Fürsorgerinnen ehrenamtlich den sog. «wilden Cliquen» zuwandten und eine Art Milieuarbeit begründeten. Mit der Machtergreifung der Nationalsozialisten fanden diese Bestrebungen allerding ihr vorläufiges Ende (vgl. Thole 2000: 130). Erst ab der zweiten Hälfte der 1960er-Jahre bemühten sich vereinzelte Initiatoren, durch ein Aufsuchen in den jeweiligen lebensweltlichen Zusammenhängen eine Brücke zu jenen Personen zu schlagen, die durch die gängigen Komm-Strukturen nicht erreicht werden konnten (vgl. Steffan 2007: 948).

\subsection{Etablierung der aSa als eigenständiges Arbeitsfeld der Sozialen Arbeit}

Nach dieser methodisch und konzeptionell eher unreflektierten Phase während der 1960er-Jahre konnte sich die aSa zwischen 1978-1981 schliesslich als genuines Arbeitsfeld der Sozialen Arbeit etablieren (vgl. Flückiger 1989: 55). Die damals entstandenen, unter den Begriffen «Gassenarbeit» oder «Streetwork» subsumierten Projekte wandten sich unterschiedlichen Zielgruppen - «Jugendbanden», Wohnungslosen, Drogenkonsumierenden usw. - zu. Im deutschen Sprachraum erfuhr die aSa im Zuge des Heroinbooms Ende der 1980er- bis Mitte der 1990er-Jahre einen nachhaltigen Entwicklungsschub (Steffan 2000: 568). Während dieser Zeitspanne wurden zahlreiche Projekte im Bereich aufsuchender Präventions- oder Betreuungsarbeit initiiert. Viele noch heute existierende aSa-Angebote lassen sich als Antwort verstehen auf nicht vorhandene Strukturen der Schadensminderung und auf die aussichtslosen Versuche von Seiten der Politik, der Drogenproblematik mit Mitteln der Repression Herr zu werden. Ein Blick auf das Aufgabengebiet der damaligen aSa des Vereins Kirchliche Gassenarbeit Luzern bestätigt diesen Befund. So schreibt zum Beispiel das aSaTeam des Vereins im Jahresbericht von 1996: «Viele Personen [...] ernähren sich nicht mehr ausgewogen und finden den Zugang zu medizinischer Versorgung erst, wenn ihr gesundheitlicher Zustand alarmierend ist. [...] Oft sind wir Zeugen, wie in den öffentlichen WC-Anlagen [...] unter erschreckenden hygienischen Bedingungen injiziert wird. In Luzern fehlt ein Gassenzimmer» (Team Gassenarbeit 1996: 12-13). Bei der Initiierung von Angeboten im Bereich Überlebenshilfe kommt der aSa ein grosses Verdienst zu. Die Schaffung von Kontakt- und Anlaufstellen, Gassenküchen, 
betreuten Wohnangeboten und Beratungsstellen für Suchtbetroffene in verschiedenen Schweizer Städten ist vielfach aus den Bestrebungen der aSa hervorgegangen. Dies gilt in besonderem Masse für die Stadt Luzern. ${ }^{2}$ Getragen wurden die aSa-Pionier-Projekte der 1980er-Jahre oftmals von kirchlichen Körperschaften, finanziert durch Zuwendungen der öffentlichen Hand.

\section{3 aSa im Spannungsfeld der Politik}

Die Existenzberechtigung einer solchen milieubezogenen aSa wurde jedoch von Beginn an von verschiedenen Seiten hinterfragt. Politische Kreise befürchteten, dass die Präsenz von Gassenarbeiterinnen und Gassenarbeitern im Bereich der offenen Drogenszene eine Auflösung derselben verhindere bzw. zu deren Stabilisierung beitrage. So geschehen in der Stadt Luzern. Dort unterhielt der Verein Kirchliche Gassenarbeit seit den Achtzigerjahren Angebote für sucht- und armutsbetroffene Menschen. Bereits in den Anfangsjahren war die aSa ein zentrales Standbein der Vereinstätigkeiten. 1993 wurde die offene Szene am Zürcher Platzspitz polizeilich geräumt, zwei Jahre später schliesslich auch jene am Letten. Dies führte unmittelbar zu einem Personenzuwachs in der offenen Szene in Luzern, namentlich an der Eisengasse. Die sich zusehends verschärfende Situation in der offenen Drogenszene Luzerns führte letztlich auch hier zur Umsetzung repressiver polizeilicher Strategien. Im Zuge dieser Entwicklungen erhöhte sich der politische Druck auf die aSa und führte zu einem Rückgang der Akzeptanz in der Bevölkerung, bis das Angebot schliesslich 1997, nach über zehnjährigem Bestehen, eingestellt werden musste. Der Verein konzentrierte sich daraufhin stärker auf ambulante Angebote innerhalb fester Einrichtungen. Im Frühling 2008 bildete sich erneut eine offene Drogenszene im Salesiapark in der Agglomerationsgemeinde Kriens. Diese neue offene Drogenszene mit teils bis zu über hundert Personen vor Ort bewegte die Gemüter, nicht zuletzt auch die politischen Kräfte. Ironischerweise gipfelte der erneute politische Diskurs in einer Volksmotion an den Stadtrat, mit exakt der gegenteiligen Stossrichtung als es das politische Klima noch vor zehn Jahre gebot: Die Motion verlangte, eine «offene und

\footnotetext{
2 Ähnliche Prozesse der Herausbildung von Suchthilfestrukturen finden sich auch in Bern, Biel oder Zürich.
} 
aufsuchende Gassenarbeit einzuführen und die dazu notwendigen rechtlichen und finanziellen Grundlagen zu schaffen» (Stadt Luzern 2009). Der Auftrag zur Einrichtung und Führung des Projektes wurde sinnigerweise dem Verein Kirchliche Gassenarbeit übertragen. Seit Oktober 2009 betreibt dieser erneut eine aSa im Einzugsgebiet der Stadt Luzern. Aufgrund der erfolgreichen Vier-Säulen-Politik haben sich die Befürchtungen und Ängste aus früheren Jahren, die aSa würde zu einer Stabilisierung offener Drogenszenen beitragen, als unbegründet erwiesen. Heute geniesst die aSa eine breite Akzeptanz in der Bevölkerung. Dementsprechend finden sich in allen grösseren Schweizer Städten etablierte und qualifiziert ausgestaltete aSa-Angebote.

\subsection{Weiterentwicklung und Professionalisierung der Disziplin in der Schweiz}

In der Schweiz haben sich die verschiedenen, im Bereich der aSa tätigen Akteure als 'Fachgruppe Gassenarbeit' (FaGass) - vormals als eine Fachgruppe des Fachverbands Sucht, heute unter dem Dach von 'Avenir Social' - zusammengeschlossen. Die Fachgruppe hat zum Ziel, den landesweiten Austausch unter den betreffenden Institutionen zu fördern. Mit der Erarbeitung von Haltungspapieren und dem Festlegen von Standards soll einerseits eine einheitliche Haltung in spezifischen Fragen geschaffen, wie auch eine stetige Professionalisierung der aufsuchenden Sozialen Arbeit vorangetrieben werden. So zeichnet sich die Fachgruppe auch verantwortlich für die Erarbeitung der 'Charta der Aufsuchenden Sozialarbeit' (vgl. FaGass 2004), ein zentrales Grundlagendokument für die schweizerische aSa. Die Charta ist in einem zweijährigen Arbeitsprozess entstanden und ist gewissermassen der «kleinstmögliche gemeinsame Nenner» (Klaus 2006: 248) der verschiedenen in der aSa tätigen Institutionen in der Schweiz. Ein weiteres, von der FaGass erarbeitetes Grundlagenpapier ist das 'Haltungspapier zu ordnungspolitischen Aufträgen', in dem die Unvereinbarkeit von Ordnungspolitik und einer professionell verstandenen aSa festgehalten wird (vgl. FaGass 2011). Die FaGass versteht sich zudem als Kompetenzzentrum für Anfragen von Externen.

Qualitätsmanagementsysteme (QMS) sind heute Bestandteil praktisch sämtlicher sozialer Institutionen, die Gelder der öffentlichen Hand 
erhalten. Das QMS 'QuaTheDA' (QualitätTherapieDrogenAlkohol) wurde 2000 vom Bundesamt für Gesundheit (BAG) für den stationären Suchthilfebereich veröffentlicht. 2006 wurde QuaTheDA auf sämtliche ambulanten und stationären Suchthilfebereiche, inklusive Schadensminderung, ausgedehnt. Auch für die aSa liegt ein eigenes Referenzsystem von QuaTheDA (Modul 8) vor, welches Qualitätsanforderungen für die Kontaktaufnahme, Beratung, Vernetzung, Konzeption und Dokumentation enthält und somit einen wichtigen Beitrag zur Professionalisierung der Teildisziplin leistet (vgl. BAG 2012: 133-141).

Ein weiteres wichtiges Element für die Weiterentwicklung und Professionalisierung ist ein entsprechendes Weiterbildungsangebot. Dieses beschränkt sich nach wie vor lediglich auf ein viertägiges Fachseminar, das von der Fachhochschule Nordwestschweiz (FHNW) angeboten wird.

\subsection{Spezifika in der Arbeitsbeziehung}

Die Notwendigkeit einer aSa wird durch die Annahme begründet, dass bei gewissen Personen wohl eine Hilfsbedürftigkeit besteht, jedoch die bestehenden Hilfs- und Beratungsangebote mit Komm-Struktur nicht in Anspruch genommen werden (können) - aus unterschiedlichen Gründen. Die Hauptursache für das vermeintliche Versagen hochschwelliger Angebote wird in einer der Personengruppe nicht adäquaten Form der Kontaktaufnahme und Beziehungsgestaltung vermutet. Dieses Defizit soll durch eine Geh-Struktur überwunden werden. In der aSa kommt es somit zu einer eigentlichen Umkehrung bei der Kontaktaufnahme zwischen AdressatInnen und Professionellen der Sozialen Arbeit: Nicht die Klientinnen und Klienten gehen auf die Sozialarbeitenden $\mathrm{zu}$, sondern werden von diesen in ihren Alltagswelten aufgesucht. Aus diesem «umgekehrten» Zugang ergeben sich eine Reihe von spezifischen Herausforderungen bzw. Fragestellungen:

1) aSa geschieht innerhalb eines konkreten geographischen Raumes eines Quartiers, Stadtteils, Platzes oder einer Strasse. Nebst der eigentlichen Zielgruppe der aSa werden diese äusseren Räume zugleich auch von zahlreichen weiteren Akteuren genutzt. Solche Räume sind immer auch Handlungsräume von Subjekten. Es sind Orte vielgestaltiger Beziehungen, Interaktionen und komplexer sozialer Zusammenhänge, was die aSa 
in ihrem Handeln stets mit zu bedenken hat. Dieser Sachverhalt verlangt nach einer sozialräumlichen Orientierung der aSa. Sozialraumorientierte Soziale Arbeit fokussiert nicht nur auf individuelle Unterstützungsprozessen wie bspw. die Einzelfallhilfe (vgl. Kessel/Reutlinger 2010: 44), sondern versucht den Veränderungswillen und die Ressourcen der Klientinnen und Klienten mit den Möglichkeiten ihres jeweiligen Sozialraums zu verbinden, z. B. Nachbarschaft, Sozialhilfe, gemeinnützige Institutionen, politische Entscheidungsträger (vgl. Klöti/Fabian/Drilling 2012: 6). ASa hat somit stets den Gesamtkontext im Blickfeld zu behalten, in dem sich ihre Adressatinnen und Adressaten bewegen und sich das eigene sozialarbeiterische Handeln abspielt.

2) Die von der aSa aufgesuchten Räume werden nebst der eigentlichen Zielgruppe auch von zahlreichen anderen Individuen und Gruppierungen genutzt, deren Forderungen und Ansprüche an den jeweiligen Raum jedoch oftmals von jenen der Zielgruppe stark divergieren. Diese abweichenden Interessen werden in der einen oder anderen Form an die Professionellen der aSa herangetragen und verlangen nach einer klaren Positionierung. Versteht sich die aSa als Anwältin ihrer Zielgruppe oder als Mediatorin zwischen den verschiedenen, den Sozialraum beanspruchenden Parteien? ASa muss sich über ihre Mandatierung im Klaren sein. Hat sie ein Doppel- oder gar ein Vielfachmandat inne? Ist eine Mehrfachmandatierung überhaupt zielführend im Kontext der aSa? In diesem Zusammenhang ist auch die Frage zu klären bzw. die Entscheidung zu treffen, ob die aSa ein für die Anspruchsgruppen bzw. Adressatinnen und Adressaten freiwilliges oder verpflichtendes Angebot sein will. Bestimmt die Zielgruppe oder bestimmen die Professionellen der aSa über Art und Dauer der Kooperation? Dabei stellt sich die Grundsatzfrage, ob überhaupt noch von aSa die Rede sein kann, wenn die Professionellen über Art und Dauer der «Kooperation» entscheiden. Das Thema der Freiwilligkeit wird weiter unten nochmals aufgegriffen.

3) Der Kontext der Arbeitsbeziehung ist im Wesentlichen geprägt durch Regeln und Codes der Zielgruppe (subkulturelle Orientierung, Milieus). So kommt es auch in dieser Hinsicht zu einer Umkehrung in der Beziehungsgestaltung: Der Rahmen der Arbeitsbeziehung wird zur Hauptsache nicht durch die Institution, sondern durch die Zielgruppe der 
aSa vorgegeben (Ort, Gesprächsdauer, Anzahl der am Gespräch beteiligten Personen, Gesprächsinhalt). Dies setzt gewisse Persönlichkeitsmerkmale bei den Professionellen der aSa voraus. So bedarf es an Selbstsicherheit und Authentizität, da Angst oder Anbiederung eine wirkungsvolle aSa verunmöglichen.

\subsection{Die Adressatinnen und Adressaten der aSa}

In zahlreichen aSa-Konzepten wird versucht, mit Hilfe soziologischer, juristischer und medizinischer Attribute wie z. B. Sucht- und Armutsbetroffenheit, Randständigkeit oder Aufenthalt im öffentlichen, respektive halböffentlichen Raum, den Adressatenkreis der aSa zu bestimmen. Die gesamtschweizerische 'Charta der Aufsuchenden Sozialarbeit' benennt «von Ausgrenzung bedrohte» (Klaus 2006: 256) Personen als Zielgruppe der aSa. Die verschiedenen aSa-BetreiberInnen grenzen die Zielgruppen dem eigenen Leitbild entsprechend ein. Der Verein Kirchliche Gassenarbeit Luzern beispielsweise definiert «sucht- und armutsbetroffene Frauen und Männer, [die] sich dauernd oder vorübergehend im öffentlichen oder halböffentlichen Raum aufhalten» (Verein Kirchliche Gassenarbeit 2018: 1) als Zielgruppe. Die Möglichkeit einer engeren, dem jeweiligen institutionellen Auftrag entsprechenden Zielgruppenbestimmung, sieht auch die Charta vor (vgl. ebd.). Wie bereits oben erwähnt, kann als eine Art Minimaldefinition festgehalten werden, dass sich aSa in ihrer Tätigkeit jenen Menschen zuwendet, deren Lebensmittelpunkt der öffentliche bzw. halböffentliche Raum ist, bei denen eine Hilfsbedürftigkeit angenommen wird, die jedoch Angebote der Sozialen Arbeit mit Komm-Struktur nicht nutzen. Eine durchaus sinnige Ausweitung dieser Minimaldefinition liefert Hochstrasser. Er benennt «Menschen, deren Lebensmittelpunkt sich auf der Gasse befindet und deren soziales Netzwerk sich weitgehend auf diesen Sozialraum reduziert hat» (Hochstrasser 2012: 12) als Adressatinnen und Adressaten der aSa und versucht so, dem 'Lebenswelt-Konzept' im Sinne Thierschs (Thiersch/Grundwald 2002: 136ff.) Rechnung zu tragen. 


\subsection{Ziele der Aufsuchenden Sozialen Arbeit}

Die Charta der Aufsuchenden Sozialarbeit hält acht zentrale Ziele von aSa fest. Diese sind:

- «die soziale Vernetzung zu fördern, zu erhalten und zu verstärken,

- zur Entwicklung, Entfaltung und Emanzipation der Individuen [...] beizutragen,

- Situationen vorzubeugen, welche die Betroffenen in ihrer physischen und/oder psychischen Unversehrtheit beeinträchtigen [...],

- beizutragen zu einer Begrenzung und Verminderung von psychischen, physischen und sozialen Beeinträchtigungen,

- [...] zu ermöglichen, gesellschaftlichem Ausschluss jeder Art zu entkommen [...] und einen verantwortungsvollen und kritischen $\mathrm{Zu}$ gang zur Gesellschaft zu finden,

- Prozesse zu unterstützen, welche die individuellen und kollektiven AkteurInnen befähigen, [...] selbstständig zu handeln,

- den Einzelnen Zugang zu benötigten Ressourcen, Dienstleistungen, Strukturen und Angeboten zu ermöglichen,

- solidarische Beziehungen und das Gefühl der Zugehörigkeit zu fördern» (Fachgruppe Gassenarbeit 2004: 11).

Auf den ersten Blick mag dies als ein sehr ehrgeiziges Programm anmuten. Tatsächlich widerspiegeln die aufgeführten Punkte sehr präzise den Arbeitsinhalt der aSa, wobei Punkt 7 (Zugang zu Ressourcen ermöglichen) einen grossen Teil der täglichen Arbeit ausmacht. Der Zielgruppe soll der Zugang zum bestehenden Hilfssystem aufgezeigt und vermittelt werden, damit sie eine angestrebte Verbesserung der Lebenssituation (Wohnsituation, Existenzsicherung, Gesundheit) erreichen kann.

\subsection{Arbeitsprinzipien und Methodik der aSa}

Wie von Hochstrasser richtig erkannt, ist die «Gasse» der Lebensmittelpunkt und Horizont der Alltagserfahrung jener Menschen, zu denen die aSa eine Brücke schlagen will. ASa vollzieht sich im Alltag ihrer Adressatinnen und Adressaten. Welche Implikationen für die Methodik und Ausgestaltung der aSa ergeben sich aus diesem nicht zu unterschätzenden 
Sachverhalt? Eine Antwort liefert Thierschs Konzept einer 'lebensweltorientierten Sozialarbeit'. Thiersch sieht die Sozialarbeit mit zahlreichen inneren Problemen konfrontiert. Er unterstellt der Profession Selbstreferentialität und eine defizitorientierte bzw. pathologisierende Sicht auf die Adressatinnen und Adressaten der Sozialen Arbeit (vgl. Thiersch/Grundwald 2002: 135). Um diese systemimmanenten Unzulänglichkeiten zu überwinden, bedient sich Thiersch - in Anlehnung an Alfred Schütz - des Begriffs des Alltags und beschreibt die Alltagserfahrung der Adressatinnen und Adressaten Sozialer Arbeit als einzig gültigen Referenzpunkt professionellen sozialarbeiterischen Handelns. Eine so verstandene lebensweltorientierte Soziale Arbeit «agiert im Horizont der radikalen Frage nach dem Sinn und der Effizienz sozialer Hilfen aus der Perspektive ihrer Adressat/innen» (ebd.). Für Thiersch folgt daraus eine Abkehr von medizinisch geprägten Hilfsformen der Sozialen Arbeit und eine Hinwendung zu den individuellen Problemlagen - und zwar so, wie diese von den Betroffenen in ihrem Alltag selbst gedeutet werden. Zielhorizont dieser lebensweltorientierten Sozialen Arbeit ist ein gelingenderer Alltag. Solchermassen verstandene und strukturierte lebensweltorientierte Hilfe ist zunächst in die sozialen Strukturen auf personaler und lokaler Ebene eingebettet, mischt sich aber auch - in anwaltlicher Funktion für die betroffenen Menschen in den sozialpolitischen Diskurs für gerechte Strukturen ein.

Die zentralen Grundgedanken der Thiersch'schen Lebensweltorientierung fanden auch Eingang in die Charta der Aufsuchenden Sozialarbeit. Dieses nach wie vor bedeutsame Grundlagendokument liefert die Basis für zahlreiche aSa-Konzepte in der Schweiz. So herrscht heute weitgehend Konsens darüber, dass aSa niederschwellig, freiwillig, parteilich, genderund diversity-gerecht, anwaltschaftlich und akzeptierend auszugestalten ist. Zudem basieren die meisten Konzepte, entsprechend der Charta, auf einer systemisch-konstruktivistischen Perspektive. Einige zentrale Prinzipien und Haltungen sollen im Folgenden kurz ausgeführt werden.

\subsubsection{Alltagsorientierung/Niederschwelligkeit}

Gerade die Arbeit mit Suchtbetroffenen, deren Lebensvollzug sich vielfach ausserhalb gängiger gesellschaftlicher Strukturen abspielt, erfordert grösstmögliche Niederschwelligkeit. Eine niederschwellige Sozialarbeit orientiert sich hinsichtlich Raum, Zeit und Methodik am Rhythmus der 
AdressatInnen (vgl. Gillich 2006: 58). Für Thiersch heisst dies, dass eine niederschwellige Sozialarbeit die bestehenden institutionellen und professionellen Zugangsbarrieren (Systemorientierung statt KlientInnenorientierung, Bürokratie, schlechte örtliche und zeitliche Erreichbarkeit, einfacher Zugang zu den Leistungen, Ausrichtung an den zeitlichen Möglichkeiten der Zielgruppe, Orientierung an den KlientInnenbedürfnisse), so weit als möglich abzubauen hat (vgl. Thiersch/Grundwald 2002: 137). Ein Angebot der aSa ist demnach so auszugestalten, dass dieses für die Zielgruppe möglichst einfach und ohne Hürden zugänglich ist. Um Hilfe in Anspruch zu nehmen, sollen möglichst keine Vorbedingungen erfüllt werden müssen. Durch eine niederschwellige aSa können die Klientinnen und Klienten in einem weiteren Schritt auch den höherschwelligen Strukturen des Hilfesystems zugeführt werden.

\subsubsection{Parteilichkeit}

Parteiliche Soziale Arbeit geht davon aus, dass «unparteiliche Arbeit» (Hartwig 2000: 14) ein Mythos ist. Das Konzept einer Parteilichen Sozialarbeit hat ihren Ursprung in der Auseinandersetzung der Sozialen Arbeit mit der Kritischen Theorie (vgl. ebd. 26). Parteiliche Sozialarbeit hinterfragt die Legitimität bestehender Machtstrukturen und ergreift klar Partei. Ihr Ziel ist es, marginalisierte, ausgegrenzte und benachteiligte Gruppen zu stärken und ihnen Gehör zu verschaffen (vgl. ebd.). Demnach hat aSa für ihre Zielgruppen Partei zu ergreifen. Dies soll aber nicht heissen, dass aSa die für professionelles, sozialarbeiterisches Handeln konstitutive Distanz innerhalb einer Hilfebeziehung aufgibt und so einem «unreflektierten Identifikationsmechanismus» (Merchel 2000: 58) Vorschub leistet. Parteilichkeit meint in diesem Kontext, dass die aSa der Tatsache Rechnung zu tragen hat, dass ihre Zielgruppe - aus welchen Gründen auch immer nicht am öffentlichen Diskurs teilhat. Sie hat sicherzustellen, dass die Anliegen der Zielgruppe wahrgenommen werden. ASa hat sich, im Interesse ihrer Adressatinnen und Adressaten, in politische Diskurse einzumischen (vgl. Thiersch 2002: 137). Für Thiersch ist eine kritisch verstandene Soziale Arbeit nur dann möglich, «wenn sie sich als sozialpolitisch versteht» (ebd. 137). 


\subsubsection{ASa und ordnungspolitische Aufträge}

Ein wachsendes Sicherheitsbedürfnis in der Bevölkerung führt in der Tendenz dazu, dass der öffentliche Raum stärker überwacht und kontrolliert wird. Es wird versucht, diesem Bedürfnis durch eine Reihe ordnungspolitischer Massnahmen Rechnung zu tragen. So wurden in den letzten Jahren in den meisten Kantonen gesetzliche Grundlagen geschaffen, die der Polizei erlauben, Personen beim Vorliegen einer Störung oder Gefährdung der öffentlichen Sicherheit wegzuweisen. ${ }^{3}$ Dies macht deutlich, dass ordnungspolitische Strategien und Massnahmen bei Nutzungskonflikten im öffentlichen Raum an Einfluss gewonnen haben (vgl. Huber 2009: 271ff.) Die seit einigen Jahren in Schweizer Städten anzutreffenden uniformierten Patrouillen können dem Bereich solcher Kontroll- bzw. ordnungspolitischer Massnahmen und Interventionen zugeordnet werden. Aus der Konsequenz einer parteilich verstandenen Sozialarbeit werden hingegen von der aSa jegliche kontroll- und ordnungspolitische Aufträge abgelehnt. Hier manifestiert sich ein fundamentaler Unterschied zwischen den Angeboten der aSa und jenen von SIP, PINTO oder ähnlichen, im öffentlichen Raum tätigen Organisationen. Diese sehen sich als allparteiliches Angebot, dessen Anliegen es ist, zu gewährleisten, dass der öffentliche Raum von unterschiedlichen Gruppen und Einzelpersonen in gleicher Weise genutzt werden kann. Sie unterscheiden sich in quantitativer Hinsicht von den sporadischen und partikularen polizeilichen Kontrollen in der Vergangenheit, da diese Patrouillen täglich durchgeführt werden. Um ihren in erster Linie ordnungspolitischen Auftrag zu erfüllen, sind SIP oder PINTO gezwungen, aktiv auf die Zielgruppe zuzugehen, auch wenn diese keinen Kontakt aufnehmen will. Anders verhält es sich mit dem freiwilligen Angebot der aSa, das von den KlientInnen angenommen oder abgewiesen werden kann. Für die Fachgruppe Gassenarbeit ist ein ordnungspolitischer Auftrag unvereinbar mit den ethischen Grundlagen der Sozialen Arbeit, was sie mit dem Verfassen eines entsprechenden Haltungspapiers zum Ausdruck brachte (vgl. Knecht (2011). Wyss (2013: 15) hat die Kontroverse umfassend dargestellt.

\footnotetext{
${ }^{3}$ Den Anfang machte der Kanton Bern mit dem sog. «Lex Wasserfallen» von 1997. In den Jahren darauf schufen praktisch sämtliche Kantone - bis auf acht - ähnliche Gesetzesgrundlagen, wobei die meisten Artikel in den Jahren 2007 und 2008 eingeführt wurden (vgl. Moeckli/Keller 2012:3f.).
} 


\subsubsection{Akzeptierende Haltung}

Über einen langen Zeitraum hinweg galt die Abstinenz als unverrückbares Dogma der Suchtarbeit (vgl. Kleinmeier 2004: 35f.). Ein solches Abstinenzparadigma greift jedoch zu kurz und lässt den Umstand ausser Acht, dass Abstinenz an sich kein Garant für eine Steigerung der Lebensqualität ist (vgl. ebd. 38). So vertreten auch die allermeisten aSa-Konzeptionen keinen Abstinenzanspruch, lehnen entsprechende Kontrollfunktionen strikte ab und setzen voraus, dass der Wunsch einer Verhaltensänderung von den Klientinnen und Klienten zu kommen hat. Im Vordergrund stehen dabei Überlegungen zur Beziehungsgestaltung und die Überzeugung, dass im Rahmen eines empathischen, vertrauensvollen und motivierenden Gesprächs mehr erreicht werden kann, als wenn der Fokus stets auf der Abhängigkeit als Defizit, das es zu überwinden gilt, liegt.

\subsubsection{Freiwilligkeit}

Mandatiert sind aSa-Mitarbeitende ausschliesslich durch ihre Klientinnen und Klienten. Doppelmandate werden ausgeschlossen. Aufträge von Dritten lassen sich demnach nicht an die aSa delegieren, da dies den Grundsätzen der Parteilichkeit, Freiwilligkeit oder zuweilen auch einer akzeptierenden Haltung widersprechen würde. Diese eindeutige Auftragslage bietet für die Professionellen der aSa einen klaren Orientierungsrahmen und vereinfacht den Zugang zur Zielgruppe. Dabei ist es jedoch zentral, dass das aSa-Mandat auch für alle anderen Stakeholder (Polizei, Bevölkerung, Politik usw.) transparent und nachvollziehbar ist - was eine intensive Kommunikation und Vernetzung der aSa mit allen Akteuren innerhalb des Sozialraumes erfordert. ASa hat sich als Teil eines Hilfesystems zu verstehen und dementsprechend «neue Formen der Kooperation» (Thiersch 2002: 137) anzustreben. Es muss ihr ein zentrales Anliegen sein, einen Beitrag an ein effektives «Zusammenspiel unterschiedlicher Kompetenzen» zu leisten - zugunsten ihrer Zielgruppe.

Es gilt an dieser Stelle darauf hinzuweisen, dass in Situationen von Fremd- und Selbstgefährdung jegliche Parteilichkeit, Freiwilligkeit oder auch akzeptierende Haltung ihre Grenzen erreicht. 


\section{Gute Praxis: aSa im Verein Kirchliche Gassenarbeit Luzern}

\subsection{Der Verein Kirchliche Gassenarbeit Luzern}

Die Tätigkeit des Vereins Kirchliche Gassenarbeit (VKG) begann mit der Einführung der aufsuchenden Sozialen Arbeit (damals Gassenarbeit) in der Stadt Luzern im Jahre 1985. Ausschlaggebend für die Einrichtung einer aSa war die sich im öffentlichen Raum manifestierende Verelendung suchtbetroffener Frauen und Männer im Zuge des Heroinbooms der 1980er-Jahre. Die römisch-katholische Kirchgemeinde Luzern reagierte auf diese Entwicklung mit der Schaffung einer 50\%-Stelle für die aSa, deren Aufgabe damals hauptsächlich im Verteilen sauberen Spritzenmaterials und der Wundversorgung bestand. Im Laufe der Jahre erweiterte sich der Tätigkeitsbereich, sodass 1993 der VKG als juristischer Verein nach ZGB Art. 60-79 gegründet wurde. Die Mitglieder des VKG sind ausschliesslich Vertreterinnen und Vertreter von Kirchgemeinden und Landeskirchen. Der Vorstand setzt sich aus den Gründungsmitgliedern des VKG zusammen. Im Rahmen der Viersäulen-Drogenpolitik des Bundes, wie sie im BetmG Art. 1a definiert ist, sind der VKG und der Verein Jobdach die einzigen Institutionen in der Säule Schadensminderung in der Stadt Luzern. Die Bereiche der Schadensminderung sind auf die beiden Vereine aufgeteilt. Während der Verein Jobdach die Bereiche Arbeit und Wohnen abdeckt, ist der VKG für die medizinische Grundversorgung, Ernährung, psychosoziale Beratung und seelsorgerliche Begleitung zuständig. Zur Zielgruppe gehören sucht- und armutsbetroffene Menschen aus dem Raum Innerschweiz. Der VKG führt die Betriebe GasseChuchi-Kontakt- und Anlaufstelle, Schalter 20 (Sozialberatung, Einkommensverwaltung), aufsuchende Sozialarbeit und Paradiesgässli (Anlaufstelle für ehemals oder aktuell suchtbetroffene Eltern mit Sozialberatung mit Einkommensverwaltung, sozialpädagogischer Familienbegleitung, Hausaufgabenhilfe und Jugendberatung). Seine Tätigkeit finanziert der VKG zu 50\% über Mittel der öffentlichen Hand, welche an Leistungsaufträge gebunden sind, zu 15\% über Beiträge der Trägerorganisationen, zu 30\% über Spenden und zu 5\% über Eigenleistungen. 


\subsection{Organisation der aufsuchenden Sozialen Arbeit}

Seit 2009 existiert erneut eine aSa. Das Angebot ist so ausgestaltet, dass an drei bis vier Abenden jeweils zwei Mitarbeitende des aSa-Teams an den für die Zielgruppe bedeutsamen Orten präsent sind. Nach verschiedenen Versuchen konnte festgestellt werden, dass ein Einsatz zwischen 17:45 bis ca. 19:45 am ergiebigsten ist, da während diesem Zeitraum sowohl die Anzahl von Menschen aus der Zielgruppe wie auch die Bereitschaft derselben, an einem Gespräch teilzunehmen, am höchsten sind. Von 2009 bis Ende 2012 bestand das aSa-Team lediglich aus Mitarbeitenden vom 'Schalter 20'. Im März 2013 wurde in dieser Hinsicht eine relevante Neuerung vorgenommen. Das aSa-Team setzt sich heute aus Mitarbeitenden sämtlicher Betriebe des VKG zusammen. Durch diese überbetriebliche Zusammensetzung kann das grosse vereinsinterne Beziehungsnetz umfassend genutzt werden. Diese Organisation der aSa erwies sich als äusserst erfolgreich, was sich einerseits an der starken Zunahme im Bereich Einkommensverwaltung als auch Sozialberatung beim Schalter 20 zeigt. Seit der Wiedereinführung der aSa haben die Einkommensverwaltungen um 100\% zugenommen. Durch die kontinuierliche Präsenz und die intensive Beziehungsarbeit ist es der aSa gelungen, bei der Zielgruppe auf die bestehenden vereinsinternen Hilfsstrukturen aufmerksam zu machen, das Vertrauen in diese zu fördern und vorhandene Hürden abzubauen.

Bei der Rekrutierung der Mitarbeitenden wird Wert auf eine qualifizierte Fach- und hohe Sozialkompetenz gelegt. Das Team besteht aus Sozialarbeitenden der Beratungsangebote, die mindestens über einen $\mathrm{Ab}$ schluss in Sozialer Arbeit (FH) verfügen, und über Mitarbeitende der GasseChuchi-K+A, die entweder einen Abschluss im Bereich der Pflege $(\mathrm{HF} / \mathrm{FH})$ oder der Sozialpädagogik/Sozialen Arbeit $(\mathrm{HF} / \mathrm{FH})$ verfügen. Weiter werden hohe Kommunikationskompetenzen sowie eine bestehende Anstellung in einem Betrieb des VKG vorausgesetzt (vgl. Verein Kirchliche Gassenarbeit 2018). Um die Fach- und Methodenkompetenz permanent zu fördern, werden zweimal jährlich interne Weiterbildungen mit externen Fachpersonen sowie regelmässige Supervisionen durchgeführt. Aktuell stehen dem VKG 90 Stellenprozente für die aSa zur Verfügung. 


\section{Fazit und Ausblick}

Wie das Beispiel des VKG zeigt, ist aSa dann wirklich effektiv, wenn sie sich mit einem ambulanten Angebot verbinden lässt. So hat sich die aSa innerhalb des VKG zu einem bedeutsamen, ja unverzichtbaren Instrument entwickelt. Einerseits gelingt es ihr, eine Brücke von den bestehenden Angeboten zu potentiellen Klientinnen und Klienten zu schlagen. Andererseits ist sie in der Lage den Interventionsrahmen traditioneller Hilfsangebote zu vergrössern und auf die Lebenswelt der Klientinnen und Klienten auszuweiten. Dies funktioniert insbesondere dann, wenn bereits eine längerfristige Zusammenarbeit mit der Klientel besteht. Ein weiterer äusserst bedeutsamer Faktor für die Wirksamkeit und Effektivität der aSa ist die Kontinuität des Angebots. Nur durch eine regelmässige Präsenz werden die Mitarbeitenden der aSa für die Adressatinnen und Adressaten auch wirklich fassbar und es kann eine Atmosphäre des Vertrauens entstehen.

Ein weiterer Erfolgsfaktor für die aSa ist ihre Innovationskraft und die Bereitschaft zur Veränderung. Die Adaption an Trends und Entwicklungen ist zwingend für einen erfolgreichen Zugang zur Klientel. So ist eine Abkehr der aSa von ihren angestammten Wirkungsfeldern (Bahnhöfe, Parks) ein durchaus realistisches Szenario. Die grossen offenen Drogenszenen der 90er-Jahre sind Geschichte. Allerdings sind neue Problemfelder erkennbar, bei deren Bearbeitung Ansatz und Methodik der aSa erfolgsversprechend erscheinen. Exemplarisch sei hier der gesamte Bereich des Nachtlebens und des Freizeitdrogenkonsums erwähnt. Die Party- und Clubkultur ist bei vielen jungen Menschen die Freizeitbeschäftigung schlechthin und oftmals auch Spielfläche risikoreichen Verhaltens. Klassischen Suchthilfestellen gelingt es kaum, diese Zielgruppe zu erreichen. Durch eine aSa, die sich szenennahen Interventionen bedient und in der Lage ist, einen wiederkehrenden Kontakt zu ihren Adressatinnen und Adressaten herzustellen, kann effektive Schadensminderung geleistet werden. Die aSa als eigenständiges Arbeitsfeld der Sozialen Arbeit ist gut beraten an ihren bewährten Arbeitsprinzipien und Grundhaltungen festzuhalten.

Die einstigen kritischen Stimmen, welche der aSa gar Szenenbildung unterstellten, sind verstummt. Die heutigen Anfragen und Herausforde- 
rungen sind gänzlich anderer Natur. Dank der vom Bund aktiv betriebenen und äusserst erfolgreichen Viersäulen-Suchtpolitik konnten zahlreiche Strukturen der Schadensminderung geschaffen werden. Die offenen Drogenszenen sind weitestgehend aus den Schweizer Städten verschwunden - und mit ihnen auch das Bewusstsein für die gesamte Suchtproblematik. Ein Blick auf den Schweizer Sorgenbarometer der Credit Suisse zeigt, dass Drogen von der Schweizer Bevölkerung heute kaum mehr als relevantes Problemfeld erachtet werden. Haben 1990 noch 70\% der Befragten Drogen als zentrales Problem wahrgenommen, sind es 2018 noch gerade 8\% (vgl. GFS 2018). Ein fehlendes Problembewusstsein in der Bevölkerung, gekoppelt mit erhöhtem Spardruck auf Seiten der aSa-Kostenträger sowie eine allgemeine Tendenz zur Medizinalisierungs, können für auch noch so erfolgreiche aSa-Projekte letztlich das Aus bedeuten.

Die aSa kommt nicht darum herum, sich mit Wirkungsforschung auseinanderzusetzen. Der Legitimierungsdruck wird kontinuierlich zunehmen. ASa hat den beabsichtigten Impact und die angestrebten gesellschaftlichen Wirkungen klar zu definieren, die entsprechenden Indikatoren und Messgrössen genau festzulegen. Nur so kann sie eine aussagekräftige Zielüberprüfung vornehmen und über ihr Tun auch auf einer soliden Basis Rechenschaft ablegen. So ist denn heute von Seiten der aSa, nebst der eigentlichen Arbeit im Feld, zunehmend eine proaktive, transparente und adressatenkonforme Kommunikation mit sämtlichen Stakeholdern sowie der Öffentlichkeit gefordert.

\section{Literatur}

Bamberger, G. (2010): Lösungsorientierte Beratung. Weinheim und Basel: Beltz Verlag. BAG - Bundesamt für Gesundheit (2012). Das modulare QuaTheDA-Referenzsystem. Die Qualitätsnorm für die Suchthilfe, Prävention und Gesundheitsförderung. Bern. BAG.

Fachgruppe Gassenarbeit (2004): Charta der Aufsuchenden Sozialarbeit. www.tinyurl.com/rx7kyyu, Zugriff 27.12.2019.

Fachgruppe Gassenarbeit (2011): Haltung zu ordnungspolitischen Aufträgen in der Aufsuchenden Sozialen Arbeit. www.tinyurl.com/v229fo3, Zugriff 27.12.2019.

Flückiger, M. (1989): Gassenarbeit in der Schweiz - ein Überblick. S. 55-60 in: W. Steffan (Hrsg.), Strassensozialarbeit - eine Methode für heisse Praxisfelder. Weinheim und Basel: Beltz.

GFS Bern (2018): Credit Suisse Sorgenbarometer. Bern: gfs. 
Gillich, S. (2006): Formen und Grundsätze der Kontaktaufnahme in den Arbeitsfeldern Streetwork und Mobile Jugendarbeit. 56-69 in: S. Gillich (Hrsg.), Professionelles Handeln auf der Strasse, Praxisbuch Streetwork und Mobile Jugendarbeit. Gründau-Rothenbergen: Triga.

Hartwig, L./Weber, M. (2000): Parteilichkeit als Konzept der Mädchen- und Frauenarbeit. S 25-48 in: L. Hartwig/J. Merchel (Hrsg.) Parteilichkeit in der Sozialen Arbeit. Münster, New York, München, Berlin: Waxmann.

Hochstrasser, T. (2013): Gassenarbeit heute. Sozial Aktuell (6): 10-13.

Huber, S. (2009). Soziale Arbeit in der Stadt - Zwischen ordnungspolitischer Vereinnahmungsversuchen und fachlichen Autonomiebestrebungen. S. 269-284 in: B. Grubenmann/J. Oelkers (Hrsg.), Das Soziale in der Pädagogik, Bad Heilbrunn: Julius Kleinhardt.

Kessel, F./Reutlinger Ch. (2010): Sozialraum, eine Einführung. Wiesbaden: VS Verlag.

Klaus, A. (2006): Blick in die Schweiz. in: S. Gillich (Hrsg.), Professionelles Handeln auf der Strasse, Praxisbuch Streetwork und Mobile Jugendarbeit. Gründau-Rothenbergen: Triga.

Kleinmeier, E. (2004): Ziele der Suchttherapie. S. 35-38 in: M. Krausz/C. Haasen (Hrsg.), Kompendium Sucht. Stuttgart, New York: Thieme.

Klöti T./Fabian, C/Drilling M. (2012): Sozialräume: verstehen - gestalten - verteidigen. SuchtMagazin 38(6): 4-11.

Knecht, R. (2011): Haltung zu ordnungspolitischen Aufträgen in der Aufsuchenden Sozialen Arbeit. Bern: Fachgruppe Aufsuchende Sozialarbeit/Streetwork von Avenir Social.

Merchel, J. (2000): Parteilichkeit: ein problematisches Prinzip für professionelles Handeln in der Sozialen Arbeit. S 49-68 in: L. Hartwig/J. Merchel (Hrsg.) Parteilichkeit in der Sozialen Arbeit. Münster, New York, München, Berlin: Waxmann.

Moeckli, D./Keller, R. (2012): Wegweisungen und Rayonverbote - ein Überblick. Sicherheit $\neg$ Recht (3): 231-245.

Specht, W. (2010): Mobile Jugendarbeit im globalen Wandel, Publikationsreihe der Internationalen Gesellschaft für Mobile Jugendarbeit, Dokumentation des 9. Internationalen ISMO Symposiums in Stuttgart.

Stadt Luzern (2009): Stellungnahme zur Volksmotion Nr. 397 2004/2009 zur Einführung der offenen und aufsuchenden Gassenarbeit.

Steffan, W. (2000): Stichwort 'Streetwork'. S. 567-574 in: F. Stimmer (Hrsg.), Suchtlexikon, München, Wien: Oldebourg.

Steffan, W. (2007): Stichwort 'Streetwork' in: R. Mulot./S. Schmitt (Hrsg.), Fachlexikon Soziale Arbeit, Baden-Baden: Nomos.

Team Gassenarbeit (1996): Weg (von) der Hoffnungslosigkeit. S. 8-16 in: Verein Kirchliche Gassenarbeit (Hrsg.), Jahresbericht 1996. Luzern: VKG.

Thiersch, H./Grunwald K. (2002): Lebenswelt und Dienstleistung. S. 67-89 in: H. Thiersch, Positionsbestimmung der Sozialen Arbeit - Gesellschaftspolitik, Theorie und Ausbildung. Weinheim und München: Juventa.

Thole, W. (2000): Kinder- und Jugendarbeit - Eine Einführung. Weinheim, München: Juventa.

Verein Kirchliche Gassenarbeit (2018): Konzept aufsuchende Sozialarbeit des Vereins Kirchliche Gassenarbeit Luzern. 
Wyss, A./Hochstrasser, T. (2012): Sozialräumliche Gassenarbeit - Anspruch oder Utopie?

SuchtMagazin 38(6): 39-42.

Wyss, A. (2013): Kontroversen in der aufsuchenden Arbeit. Sozial Aktuell (6): 14-15.

Open Access Dieses Kapitel wird unter der Creative Commons Namensnennung 4.0 International Lizenz (http://creativecommons.org/licenses/by/4.0/deed.de) veröffentlicht, welche die Nutzung, Vervielfältigung, Bearbeitung, Verbreitung und Wiedergabe in jeglichem Medium und Format erlaubt, sofern Sie den/die ursprünglichen Autor(en) und die Quelle ordnungsgemäß nennen, einen Link zur Creative Commons Lizenz beifügen und angeben, ob Änderungen vorgenommen wurden.

Die in diesem Kapitel enthaltenen Bilder und sonstiges Drittmaterial unterliegen ebenfalls der genannten Creative Commons Lizenz, sofern sich aus der Abbildungslegende nichts anderes ergibt. Sofern das betreffende Material nicht unter der genannten Creative Commons Lizenz steht und die betreffende Handlung nicht nach gesetzlichen Vorschriften erlaubt ist, ist für die oben aufgeführten Weiterverwendungen des Materials die Einwilligung des jeweiligen Rechteinhabers einzuholen.

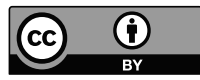

